\title{
COMPARISON THEOREMS FOR ELLIPTIC EQUATIONS ON UNBOUNDED DOMAINS
}

\author{
BY \\ C. A. SWANSON( $\left.{ }^{1}\right)$
}

Comparison theorems of Sturm's type will be obtained for the linear elliptic partial differential equations

$$
\begin{gathered}
l u=\sum_{i, j=1}^{n} D_{i}\left(a_{i j} D_{j} u\right)+2 \sum_{i=1}^{n} b_{i} D_{i} u+c u=0, \\
L v=\sum_{i, j=1}^{n} D_{i}\left(A_{i j} D_{j} v\right)+2 \sum_{i=1}^{n} B_{i} D_{i} v+C v=0
\end{gathered}
$$

on unbounded domains $R$ in $n$-dimensional Euclidean space $E^{n}$. The boundary $P$ of $R$ is supposed to have a piecewise continuous unit normal vector at each point. Points in $E^{n}$ are denoted by $x=\left(x^{1}, x^{2}, \ldots, x^{n}\right)$ and differentiation with respect to $x^{i}$ is denoted by $D_{i}, i=1,2, \ldots, n$. The coefficients $a_{i j}, b_{i}, c, A_{i j}, B_{i}$, and $C$ in (1) and (2) are assumed to be real and continuous on $R \cup P$, and the matrices $\left(a_{i j}\right)$ and $\left(A_{i j}\right)$ symmetric and positive definite in $R$. A "solution" of (1) (or (2)) is supposed to be continuous in $R \cup P$ and have uniformly continuous first partial derivatives in $R$, and all derivatives involved in (1) (or (2)) are supposed to exist, be continuous, and satisfy the differential equation at every point in $R$.

Some recent results of Clark and the author [2], [7] apply to bounded domains $R$ in $E^{n}$. In the self-adjoint case $b_{i}=B_{i}=0, i=1,2, \ldots, n$, the variation of $l u$ is defined as

$$
V[u]=\int_{R}\left[\sum_{i, j=1}^{n}\left(a_{i j}-A_{i j}\right) D_{i} u D_{j} u+(C-c) u^{2}\right] d x .
$$

The following result [2] is typical of those to be extended to unbounded domains.

THEOREM A. Let $R$ be a bounded domain in $E^{n}$ whose boundary $P$ has a piecewise continuous unit normal. Suppose $b_{i}=B_{i}=0$ in (1), (2), $i=1,2, \ldots, n$. If there exists a nontrivial solution $u$ of $l u=0$ in $R$ such that $u=0$ on $P$ and $V[u] \geqq 0$, then every solution of $L v=0$ vanishes at some point in $\bar{R}$.

In [7] the author extended this to the general second-order linear elliptic equations (1) and (2). Theorem A generalizes a theorem of Hartman and Wintner [4] in which the condition $V[u] \geqq 0$ is replaced by the pointwise conditions $C \geqq c$ and $\left(a_{i j}-A_{i j}\right)$ is positive semidefinite in $\bar{R}$. In the case $n=1$, Theorem $\mathrm{A}$ reduces to

Presented to the Society, August 30, 1966; received by the editors July 13, 1966.

( $\left.{ }^{1}\right)$ This research was supported by the United States Air Force Office of Scientific Research under grant AF-AFOSR-379-66. 
Leighton's generalization [5] of the classical Sturm-Picone theorem. In this case, because the solutions of second order ordinary linear differential equations have only simple zeros, it is easy to obtain the following modification of Theorem A: If there exists a nontrivial solution of $l u=0$ in $(\alpha, \beta)$ such that $u(\alpha)=u(\beta)=0$ and $V[u]>0$, then every solution of $L v=0$ has a zero in $(\alpha, \beta)$. In the case $n=2$, Protter [6] obtained pointwise conditions on the coefficients in (1) and (2) to ensure the conclusion of Theorem A.

Our purpose here is to extend Theorem A to unbounded domains in $E^{n}$. Apparently no general results are known even in the case $n=1$. Our results will constitute an extension of the Sturm-Picone theorem in 4 directions: (i) to $n$-dimensions; (ii) to nonselfadjoint differential equations; (iii) to coefficients satisfying a general condition of the type $V[u] \geqq 0$; and (iv) to unbounded domains.

However, differential equations of order higher than 2 and general boundary conditions will not be considered here.

Let $D_{a}$ denote the $n$-disk $\left\{x \in E^{n}:\left|x-x_{0}\right|<a\right\}$ and let $S_{a}$ denote the bounding $(n-1)$-sphere, where $x_{0}$ is a fixed point in $E^{n}$. Define

$$
R_{a}=R \cap D_{a}, \quad P_{a}=P \cap D_{a}, \quad C_{a}=R \cap S_{a} .
$$

Clearly there exists a positive number $a_{0}$ such that $R_{a}$ is a bounded domain with boundary $P_{a} \cup C_{a}$ for all $a \geqq a_{0}$.

Let $Q[z]$ be the quadratic form in $n+1$ variables $z_{1}, z_{2}, \ldots, z_{n+1}$ defined by

$$
Q[z]=\sum_{i, j=1}^{n} A_{i j} z_{i} z_{j}-2 z_{n+1} \sum_{i=1}^{n} B_{i} z_{i}+G z_{n+1}^{2},
$$

where the continuous function $G$ is to be determined so that this form is positive semidefinite. The matrix $Q$ associated with $Q[z]$ has the block form

$$
Q=\left(\begin{array}{cr}
A & -B \\
-B^{T} & G
\end{array}\right), \quad A=\left(A_{i j}\right),
$$

where $B^{T}$ is the $n$-vector $\left(B_{1}, B_{2}, \ldots, B_{n}\right)$. Let $B_{i}^{*}$ denote the cofactor of $-B_{i}$ in $Q$. Since $A$ is positive definite, a necessary and sufficient condition for $Q$ to be positive semidefinite is det $Q \geqq 0$, or

$$
G \operatorname{det}\left(A_{i j}\right) \geqq-\sum_{i=1}^{n} B_{i} B_{i}^{*} .
$$

The proof is a slight modification of the well-known proof for positive definite matrices [3].

Let $M_{a}$ be the quadratic functional defined by

where

$$
M_{a}[u]=\int_{R_{a}} F[u] d x
$$

$$
F[u]=\sum_{i, j} A_{i j} D_{i} u D_{j} u-2 u \sum_{i} B_{i} D_{i} u+(G-C) u^{2}
$$


Define $M[u]=\lim _{a \rightarrow \infty} M_{a}[u]$ (whenever the limit exists). The domain $\mathfrak{D}_{M}$ of $M$ is defined to be the set of all real-valued continuous functions $u$ in $R \cup P$ such that $u$ has uniformly continuous first partial derivatives in $R_{a}$ for all $a \geqq a_{0}, M[u]$ exists, and $u$ vanishes on $P$. Define

$$
[u, v]_{a}=\int_{C_{a}} u \sum_{i, j} A_{i j} n_{i} D_{j} v d s
$$

where $\left(n_{i}\right)$ is the unit normal to $C_{a}$;

$$
[u, v]=\lim _{a \rightarrow \infty}[u, v]_{a}
$$

whenever the limit on the right side exists.

Lemma 1. Suppose $G$ satisfies (5) in $R$. If there exists $u \in \mathfrak{D}_{M}$ not identically zero such that $M[u]<0$, then every solution $v$ of $L v=0$ for which $\left[u^{2} / v, v\right] \geqq 0$ vanishes at some point of $R \cup P$.

Proof. Suppose to the contrary that there exists a solution $v \neq 0$ in $R \cup P$. For $u \in \mathfrak{D}_{M}$ define

$$
\begin{aligned}
X^{i} & =v D_{i}(u / v), \\
Y^{i} & =v^{-1} \sum_{j} A_{i j} D_{j} v, \quad i=1,2, \ldots, n .
\end{aligned}
$$

The following identity in $R$ will now be established:

$$
\sum_{i, j} A_{i j} X^{i} X^{j}-2 u \sum_{i} B_{i} X^{i}+G u^{2}+\sum_{i} D_{i}\left(u^{2} Y^{i}\right)=F[u]+u^{2} v^{-1} L v .
$$

The left member of $(10)$ is equal to

$$
\begin{aligned}
\frac{1}{v^{2}} \sum_{i, j} A_{i j}\left(v D_{i} u-u D_{i} v\right)\left(v D_{j} u-u D_{j} v\right)-\frac{2 u}{v} \sum_{i} B_{i}\left(v D_{i} u-u D_{i} v\right) \\
+G u^{2}+\frac{2 u}{v} \sum_{i, j} A_{i j} D_{i} u D_{j} v+\frac{u^{2}}{v^{2}} \sum_{i, j}\left(v D_{i}\left(A_{i j} D_{j} v\right)-A_{i j} D_{i} v D_{j} v\right) .
\end{aligned}
$$

Since $\left(A_{i j}\right)$ is symmetric, this reduces easily to the right member of (10). Since $L v=0$ in $R$,

$$
\int_{R_{a}} F[u] d x=\int_{R_{a}}\left[\sum_{i, j} A_{i j} X^{i} X^{j}-2 u \sum_{i} B_{i} X^{i}+G u^{2}\right] d x+\int_{R_{a}} \sum_{i} D_{i}\left(u^{2} Y^{i}\right) d x .
$$

The first integrand on the right side is a positive semidefinite form by the hypothesis (5). Since $u=0$ on $P_{a}$, it follows from Green's formula that

$$
\begin{aligned}
\int_{R_{a}} \sum_{i} D_{i}\left(u^{2} Y^{i}\right) d x & =\int_{P_{a} \cup C_{a}} \sum_{i} u^{2} n_{i} Y^{i} d s \\
& =\int_{C_{a}} \frac{u^{2}}{v} \sum_{i, j} A_{i j} n_{i} D_{j} v d s .
\end{aligned}
$$


Hence (8) and (11) yield

$$
\int_{R_{a}} F[u] d x \geqq\left[u^{2} / v, v\right]_{a} .
$$

Since $\left[u^{2} / v, v\right] \geqq 0$ by hypothesis,

$$
M[u]=\lim _{a \rightarrow \infty} \int_{R_{a}} F[u] d x \geqq 0 .
$$

This contradiction establishes Lemma 1.

LEMMA 2 (SELF-ADJOINT CASE). Suppose $B_{i}=0$ in (2) and (7), $i=1,2, \ldots, n$. If there exists $u \in \mathfrak{D}_{M}$ not identically zero such that $M[u] \leqq 0$, then every solution $v$ of $L v=0$ for which $\left[u^{2} / v, v\right] \geqq 0$ vanishes at some point of $R \cup P$.

Proof. In this case we can take $G=0$, and the first integrand on the right side of (11) is a positive definite form. Hence

$$
\int_{R_{a}} \sum_{i, j} A_{i j} X^{i} X^{j} \geqq 0,
$$

equality holding iff $X^{i}$ is identically zero for each $i=1,2, \ldots, n$; i.e., $u$ is a constant multiple of $v$. The latter cannot occur since $u=0$ on $P$ and $v \neq 0$ on $P$, and therefore

$$
\int_{R_{a}} F[u] d x>\left[u^{2} / v, v\right]_{a} .
$$

It follows that $M[u]>0$, contrary to the hypothesis $M[u] \leqq 0$.

In addition to (6) consider the quadratic functional defined by

$$
m_{a}[u]=\int_{R_{a}}\left[\sum_{i, j} a_{i j} D_{i} u D_{j} u-2 u \sum_{i} b_{i} D_{i} u-c u^{2}\right] d x,
$$

whose Euler-Jacobi operator is $l$ and let $m[u]=\lim _{a \rightarrow \infty} m_{a}[u]$ (whenever the limit exists). The domain $\mathfrak{D}_{m}$ of $m$ consists of all real-valued continuous functions $u$ in $R \cup P$ such that $u$ has uniformly continuous first partial derivatives in $R_{a}$ for all $a \geqq a_{0}, m[u]$ exists, and $u$ vanishes on $P$. The variation of $m[u]$ is defined as $V[u]=m[u]-M[u]$, that is

$$
V[u]=\int_{R}\left[\sum_{i, j}\left(a_{i j}-A_{i j}\right) D_{i} u D_{j} u-2 u \sum_{i}\left(b_{i}-B_{i}\right) D_{i} u+(C-c-G) u^{2}\right] d x
$$

with domain $\mathfrak{D}=\mathfrak{D}_{m} \cap \mathfrak{D}_{M}$. The analogues of (8), (9) for the operator $l$ are

$$
\begin{aligned}
\{u, v\}_{a} & =\int_{C_{a}} u \sum_{i, j} a_{i j} n_{i} D_{j} v d s, \\
\{u, v\} & =\lim _{a \rightarrow \infty}\{u, v\}_{a} .
\end{aligned}
$$

THEOREM 1. Suppose $G$ satisfies (5). If there exists a nontrivial solution $u \in \mathfrak{D}$ of $l u=0$ such that $\{u, u\} \leqq 0$ and $V[u]>0$, then every solution $v$ of $L v=0$ for which 
$\left[u^{2} / v, v\right] \geqq 0$ vanishes at some point of $R \cup P$. The same conclusion holds if the conditions $V[u]>0,\left[u^{2} / v, v\right] \geqq 0$ are replaced by $V[u] \geqq 0,\left[u^{2} / v, v\right]>0$ respectively.

Proof. Since $u=0$ on $P_{a}$, it follows from Green's formula that

$$
m_{a}[u]=-\int_{R_{a}} u l u d x+\{u, u\}_{a} .
$$

Since $l u=0$ and $\{u, u\} \leqq 0$, we obtain in the limit $a \rightarrow \infty$ that $m[u] \leqq 0$. The hypothesis $V[u]>0$ is equivalent to $M[u]<m[u]$. Hence the condition $M[u]<0$ of Lemma 1 is fulfilled and $v$ vanishes at some point of $R \cup P$. The second statement of Theorem 1 follows upon obvious modification of the inequalities.

THEOREM 2 (SELF-ADJOINT CASE). Suppose $b_{i}=B_{i}=0$ in (1) and (2), $i=1,2, \ldots, n$. If there exists a nontrivial solution $u \in \mathfrak{D}$ of $l u=0$ such that $\{u, u\} \leqq 0$ and $V[u] \geqq 0$, then every solution $v$ of $L v=0$ for which $\left[u^{2} / v, v\right] \geqq 0$ vanishes at some point of $R \cup P$.

This follows from Lemma 2 by a proof analogous to that of Theorem 1 .

In the case that equality holds in (5), that is

$$
G=-\sum_{i=1}^{n} B_{i} B_{i}^{*} / \operatorname{det}\left(A_{i j}\right),
$$

we define

$$
\delta=\sum_{i=1}^{n} D_{i}\left(b_{i}-B_{i}\right)+C-c-G .
$$

It follows from (12) by partial integration that

$$
V[u]=\int_{R}\left[\sum_{i, j}\left(a_{i j}-A_{i j}\right) D_{i} u D_{j} u+\delta u^{2}\right] d x+\Omega,
$$

where

$$
\Omega=\lim _{a \rightarrow \infty} \int_{C_{a}} \sum_{i}\left(B_{i}-b_{i}\right) u^{2} n_{i} d s .
$$

$L$ is called a "strict Sturmian majorant" of $l$ when the following conditions hold: (i) $\left(a_{i j}-A_{i j}\right)$ is positive semidefinite and $\delta \geqq 0$ in $R$; (ii) $\Omega \geqq 0$; and (iii) either $\delta>0$ at some point in $R$ or $\left(a_{i j}-A_{i j}\right)$ is positive definite and $c \neq 0$ at some point. A function defined in $R$ is said to be of class $C^{2,1}(R)$ when all of its second partial derivatives exist and are Lipschitzian in $R$.

THEOREM 3. Suppose $L$ is a strict Sturmian majorant of $l$ and all the coefficients $a_{i j}$ involved in $l$ are of class $C^{2,1}(R)$. If there exists a nontrivial solution $u \in D$ of $l u=0$ such that $\{u, u\} \leqq 0$, then every solution $v$ of $L v=0$ for which $\left[u^{2} / v, v\right] \geqq 0$ vanishes at some point of $R \cup P$.

Proof. $V[u]$ exists since $u \in \mathfrak{D}$, and hence each term on the right side of (13) 
exists by the strict Sturmian hypothesis. Since $a_{i j} \in C^{2,1}(R), i, j=1,2, \ldots, n$, Aronszajn's unique continuation theorem [1] guarantees that the nontrivial solution $u$ cannot vanish identically in any open subset of $R$. In the case that $\delta>0$ at some point in $R$ it then follows from (13) that $V[u]>0$. In the case that $\delta \equiv 0$ in $R$ it follows from (13) and the positive definite hypothesis on $\left(a_{i j}-A_{i j}\right)$ that $V[u]=0$ only if $D_{i} u=0$ for each $i=1,2, \ldots, n$ in some open set $S$ of $R$, that is, $u$ is constant in $S$. Since $c \neq 0$ at some point $x_{0} \in S$, the differential equation (1) would not be satisfied at $x_{0}$. Hence $V[u]>0$ also in the case that $\delta \equiv 0$. The conclusion of Theorem 3 then follows from Theorem 1.

TheOREM 4 (SELF-ADJOINT CASE). Suppose $b_{i}=B_{i}=0$ in (1) and (2), $i=1,2, \ldots, n$, $C \geqq c$, and $\left(a_{i j}-A_{i j}\right)$ is positive semidefinite in $R \cup P$. If there exists a nontrivial solution $u \in \mathfrak{D}$ of (1) such that $\{u, u\} \leqq 0$, then every solution $v$ of (2) for which $\left[u^{2} / v, v\right] \geqq 0$ vanishes at some point of $R \cup P$.

This is an immediate consequence of Theorem 2 . We assert that the same conclusion holds even if $\left(A_{i j}\right)$ is only positive semidefinite, provided $L$ is a strict Sturmian majorant of $l$ and all the coefficients $a_{i j}$ are of class $C^{2,1}(R)$. In fact, under these assumptions $V[u]>0$ as in Theorem 3 , i.e., $M[u]<0$ by the proof of Theorem 1, and Lemma 2 remains valid for positive semidefinite $\left(A_{i j}\right)$ provided the hypothesis $M[u] \leqq 0$ is replaced by $M[u]<0$.

With trivial modifications the above theorems and proofs remain valid in the case that $R$ is a bounded domain, i.e., $C_{a}$ is void for $a \geqq a_{0}$. In particular Theorem 2 implies Theorem A and Theorem 1 implies the author's recent result [7] for the general elliptic equations (1), (2) on bounded domains.

In the case $n=2$ considered by Protter [6], the condition $\delta \geqq 0$ of Theorem 3 reduces to

$$
\left(A_{11} A_{22}-A_{12}^{2}\right)\left(\sum_{i=1}^{2} D_{i}\left(b_{i}-B_{i}\right)+C-c\right) \geqq A_{11} B_{2}^{2}-2 A_{12} B_{1} B_{2}+A_{22} B_{1}^{2} .
$$

If $R$ is a bounded domain, Theorem 3 then reduces (with trivial modifications) to the author's result in [7].

It is interesting to note the following one-dimensional instances of Theorem 2 , in which $R$ is an open interval $(\alpha, \beta)$. When $n=1$ and $b_{1}=B_{1}=0$, the differential equations (1), (2) have the form

$$
\begin{aligned}
\left(a u^{\prime}\right)^{\prime}+c u & =0, & & a>0, \\
\left(A v^{\prime}\right)^{\prime}+C v & =0, & & A>0 .
\end{aligned}
$$

THEOREM 5. If there exists a nontrivial solution $u$ of $(14)$ in $(\alpha, \infty)$ such that $u(\alpha)=0, a(x) u(x) u^{\prime}(x) \rightarrow 0$ as $x \rightarrow \infty$, and

$$
\int_{\alpha}^{\infty}\left[(a-A) u^{\prime 2}+(C-c) u^{2}\right] d x \geqq 0,
$$


then every solution $v$ of $(15)$ for which $A(x) u^{2}(x) v^{\prime}(x) / v(x)$ has a nonnegative limit as $x \rightarrow \infty$ has a zero on $[\alpha, \infty)$. Unless $v$ is a constant multiple of $u$, $v$ has a zero in $(\alpha, \infty)$.

Proof. The first statement follows immediately from Theorem 2. To prove the second statement, recall from the proof of Lemma 1 that for all $a \geqq a_{0}$,

$$
\int_{\alpha}^{a} F[u] d x=\left[\frac{A(x) u^{2}(x) v^{\prime}(x)}{v(x)}\right]_{\alpha}^{a}+\int_{\alpha}^{a} A v^{2}\left(\frac{u}{v}\right)^{\prime 2} d x .
$$

Since the solutions of second order ordinary linear differential equations have only simple zeros, an application of L'Hospital's rule yields

$$
\lim _{x \rightarrow \alpha} \frac{A(x) u^{2}(x) v^{\prime}(x)}{v(x)}=0 .
$$

Thus the limit of the first term on the right side of (17) as $a \rightarrow \infty$ is nonnegative. The second term is nonnegative for all $a$ and zero iff $u$ is a constant multiple of $v$. Hence $M[u]>0$ unless $v$ is a constant multiple of $u$. This contradicts the hypothesis (16).

The next result applies to the case that $\alpha, \beta$ may be singular points of the differential equations (14), (15); the possibility that they are $\pm \infty$ is not excluded. The proof is similar to that of Theorem 5 and will be omitted.

THEOREM 6. If there exists a nontrivial solution $u$ of $(14)$ in $(\alpha, \beta)$ such that $a(x) u(x) u^{\prime}(x) \rightarrow 0$ as $x \rightarrow \alpha$ and as $x \rightarrow \beta$, and

$$
\int_{\alpha}^{\beta}\left[(a-A) u^{\prime 2}+(C-c) u^{2}\right] d x>0,
$$

then every solution $v$ of $(15)$ for which $A(x) u^{2}(x) v^{\prime}(x) / v(x)$ has a nonnegative limit as $x \rightarrow \beta$ and a nonpositive limit as $x \rightarrow \alpha$ has a zero in $(\alpha, \beta)$. If the left side of $(18)$ is only nonnegative, the same conclusion holds unless $v$ is a constant multiple of $u$.

In the special case that $\alpha, \beta$ are ordinary points of (14) and (15), this reduces to the following generalization of the classical Sturm-Picone theorem; our result is a slight extension of Leighton's theorem [5].

THEOREM 7. If there exists a nontrivial solution $u$ of $(14)$ in $[\alpha, \beta]$ such that $u(\alpha)=u(\beta)=0$ and the left side of (18) is nonnegative, then every solution of (15) except a constant multiple of $u$ has a zero in $(\alpha, \beta)$.

As an example of Theorem 5, consider the differential equations

$$
\begin{aligned}
u^{\prime \prime}+\left(2 n+1-x^{2}\right) u & =0, \\
v^{\prime \prime}+\left[2 n+1-x^{2}+p(x)\right] v & =0,
\end{aligned}
$$

on a half-open interval $[\alpha, \infty)$, where $p(x)$ is a polynomial. Equation (19) has the well-known solution $u(x)=\exp \left(-x^{2} / 2\right) H_{n}(x)$, where $H_{n}(x)$ denotes the Hermite 
polynomial of degree $n$. Clearly $u \in \mathfrak{D}$. Since every solution $v$ of (20) satisfies $v^{\prime}(a) / v(a) \sim q(a)$ as $a \rightarrow \infty$, where $q(a)$ is a polynomial, it follows that the hypothesis $u^{2}(a) v^{\prime}(a) / v(a) \rightarrow 0$ as $a \rightarrow \infty$ is fulfilled. Hence if $\alpha$ is a zero of $H_{n}(x)$, then every solution of $(20)$ has a zero in $(\alpha, \infty)$ provided

$$
\int_{\alpha}^{\infty} p(x) u^{2}(x) d x>0 \text {. }
$$

\section{REFERENCES}

1. N. Aronszajn, A unique continuation theorem for solutions of elliptic partial differential equations or inequalities of second order, J. Math. Pures Appl. 36 (1957), 235-249.

2. Colin Clark and C. A. Swanson, Comparison theorems for elliptic differential equations, Proc. Amer. Math. Soc. 16 (1965), 886-890.

3. F. R. Gantmacher, The theory of matrices, Vol. I, Chelsea, New York, 1959.

4. Philip Hartman and Aurel Wintner, On a comparison theorem for self-adjoint partial differential equations of elliptic type, Proc. Amer. Math. Soc. 6 (1955), 862-865.

5. Walter Leighton, Comparison theorems for linear differential equations of second order, Proc. Amer. Math. Soc. 13 (1962), 603-610.

6. M. H. Protter, A comparison theorem for elliptic equations, Proc. Amer. Math. Soc. 10 (1959), 296-299.

7. C. A. Swanson, A comparison theorem for elliptic differential equations, Proc. Amer. Math. Soc. 17 (1966), 611-616.

UNIVERSity OF British Columbia, Vancouver, B. C., Canada 